\title{
Potential benefits of incorporating energy information when estimating attenuation from PET data
}

\author{
Ludovica Brusaferri, Student Member, IEEE, Alexandre Bousse, Nikos Efthimiou Member, IEEE, \\ Elise Emond, Student Member, IEEE, David Atkinson, Sébastien Ourselin, Brian F. Hutton, Simon Arridge and \\ Kris Thielemans, Senior Member, IEEE
}

\begin{abstract}
This study explores the advantages of using a different energy window for each individual annihilation pho or when estimating attenuation from scattered PET data. Sevía studies have shown that scattered data can be used to reconstruct the density distribution of the object, although they are limited to simple phantoms and ideal energy measurements. We have incorporated a realistic photon detection probability model into our algorithm, as well as the dependence of attenuation on photon energy. In the proposed method each detector can assign a photon to either energy window. Preliminary results on point source simulations have shown that using a different window for each annihilation photon allows to derive spatial information on both attenuation and activity distribution, and to isolate subsets of scatter angles depending on the energy window pair used. XCAT simulations demonstrated that it is possible to yield an attenuation map from scattered PET data only and that MR information can increase reconstruction accuracy. This method can also compensate for errors typically introduced from the MRAC, such as truncation artifacts.
\end{abstract}

\section{INTRODUCTION}

A TTENUATION correction represents one of the major challenges to be faced in hybrid PET/MRI systems, as the MR signal is not directly correlated to tissue attenuation.

Several attempts have been made to estimate attenuation coefficients solely derived from the PET emission data, including

Manuscript received November 10, 2017. This work is supported by Siemens Healthineers, the UCL Impact Scheme, NIHR UCLH BRC, EPSRCfunded UCL Centre for Doctoral Training in Medical Imaging (EP/L016478/1) and GlaxoSmithKline (BIDS3000030921)

Ludovica Brusaferri is with the Institute of Nuclear Medicine, University College London, London NW1 2BU, UK (e-mail: 1.brusaferri.16@ucl.ac.uk).

Alexandre Bousse is with the Institute of Nuclear Medicine, University

College London, London NW12BU, UK (e-mail: a.bousse@ucl.ac.uk).

Nikos Efthimiou is with the School of Life Sciences, Faculty of Health Sciences, University of Hull, Hull HU6 7RX, UK (e-mail: n.efthymiou@hull.ac.uk).

Elise Emond is with the Institute of Nuclear Medicine, University College London, London NW12BU, UK (e-mail: elise.emond.16@ucl.ac.uk).

David Atkinson is with the Centre for Medical Imaging, University College London, London NW1 2HE, UK (e-mail: a.atkinson@ucl.ac.uk).

Sébastien Ourselin is with the Department of Medical Physics and Biomedical Engineering, University College London, London WC1E 6BT, UK (e-mail: s.ourselin@ucl.ac.uk).

Brian Hutton is with the Institute of Nuclear Medicine, University College London, London NW1 2BU, UK (e-mail: b.hutton@ucl.ac.uk).

Simon Arridge is with the Department of Computer Science, University College London, London WC1E 6BT, UK (e-mail: S.Arridge@cs.ucl.ac.uk).

Kris Thielemans is with the Institute of Nuclear Medicine, University College London, London NW1 2BU, UK (e-mail: k.thielemans@ucl.ac.uk). the MLAA approach proposed by Nuyts et al._ [1], known as Ic kimum likelihood reconstruction of attenuation and activity (MLAA) . Nevertheless, reconstruction algorithms that only consider coincidences detected with a conventional energy window, and without the availability of time-of-flight (TOF), suffer from cross-talk between attenuation and emission data resulting from reconstruction, and a scaling problem between the reconstructed and the true activity distribution [2].

Motivated by the fact that attenuation in PET is mainly due to Compton scattering, and that scatter events represent up to $40 \%$ of the total recorded PET coincidences [3], several groups have investigated the possibility of using scattered data as an additional source of information to overcome reconstruction ambiguities [4], [5]. These attempts share common roots with SPECT studies, and extend earlier suggestion of using individual photon energies [6].

Although scattered data have been successfully used to reconstruct attenuation map in SPECT [7], [8], this is still at an early stage of development in PET. Current techniques have shown good results with point-sources and simple 2D phantoms, and rely on good-to-perfect energy resolution [9][12].

The idea of deriving quantitative information from scatter relies on the possibility of estimating scattering angles through photon energy measurements. This explains why energy uncertainty represents one of the main limitations when dealing with scattered data, since trace back to the scatter angle given the detected energy is straightforward only if energy measurements are perfect.

In this work we therefore aim to demonstrate the benefits of using a different energy window for each individual annihilation photon to perform attenuation estimation when dealing with realistic energy measurements.

The current study is restricted to single scatter events.

\section{METHOD}

\section{A. Energy Based Single Scatter Model}

According to the Single Scatter Simulation (SSS) algorithm [13], given two detectors $A$ and $B$ and a scatter point $S$, two different contributions must be considered when evaluating the expected number of scattered counts, depending on which side of the scatter point the emission point lies (1). 


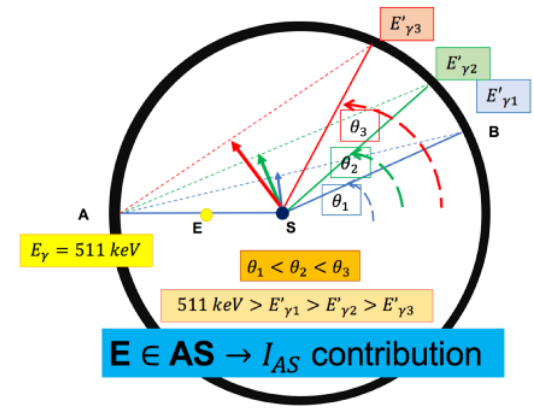

Fig. 1. Scattered coincidence with emitter located on AS side.

$$
I_{L O R, S}=I_{* A S}+I_{* B S}
$$

When a scattered coincidence with the emitter located on AS side occurs (Fig. 1), the scattered photon is detected at detector A. Detector B, instead, collects the scattered photon, which has a lower energy $\mathrm{E} \gamma$, that depends on the scattered angle $\theta_{S}$. The reverse situation arises when emitters are located on BS side.

Considering that these two events can be discriminated based on the individual energy of the photons, measuring both photon energies with the same energy window is suboptimal.

Ideally, to have the highest probability of detection, and therefore the highest amount of counts, one would want to measure the event shown in Fig. 1 with a lower energy window in $\mathrm{B}$ and an upper energy window in A. We will refer to this case as UL, and vice versa as LU. Furthermore, the UU notation corresponds to the situation when the two photons are detected with the same upper energy window, and LL with the lower one.

Each of the four configurations provides different information. To investigate this, we extended the SSS model to a multiple energy window scenario, assuming that each detector can assign a photon to either energy window.

As a result, for a given $\mathrm{LOR}_{A B}$ and an energy window pair $w, y$, the total amount of expected scatter counts is given by:

$$
\begin{gathered}
\bar{g}_{w y}(\lambda, \mu)=\sum_{n=1}^{N_{S}}\left\{\epsilon_{w}(511) \epsilon_{y}\left(E\left(\theta_{S_{n}}\right)\right) I_{* A S_{n} B}+\right. \\
\left.\epsilon_{w}\left(E\left(\theta_{S_{n}}\right)\right) \epsilon_{y}(511) I_{A S_{n} * B}\right\}
\end{gathered}
$$

where $\epsilon$ is the detection probability, $N_{S}$ is the total number of scatter points, $w$ and $y$ are the energy windows and $\theta_{S}$ is the scatter angle.

The detection probability has been modeled as a Gaussian function $\epsilon=f(E, r, e w)$, which depends on incident photon energy $E$, energy resolution $r$ and energy window ew.

Software implementing this model was written, based on the scatter implementation in STIR [14].

\section{B. Forward Model Investigation - Preliminary Study}

In order to investigate what kind of information can be derived from each sinogram, we did a preliminary study on a

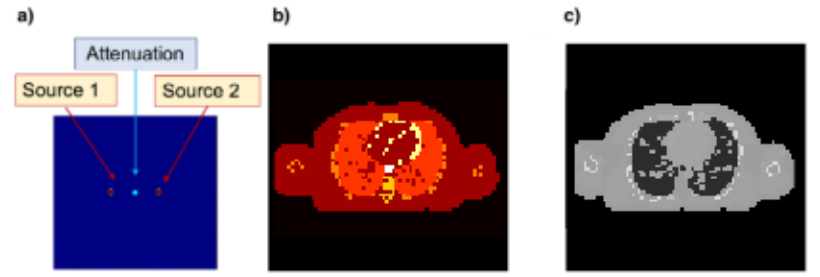

Fig. 2. From left to right: point-source phantom (a), XCAT emission image (b), XCAT attenuation image (c)

simple phantom made of two identical point-source emitters, symmetric with respect to the attenuation source, as shown in Fig.2a. XCAT [15] phantom (Fig.2b,2c) simulations were used to investigate outcomes for more realistic imaging data. XCAT images used were $77 \times 77 \times 15$ with a voxel dimension of $0.6258 \times 0.6258 \times 0.675 \mathrm{~cm}^{3}$.

Simulations have been performed by using Siemens ECAT 931 and Siemens mMR scanner geometric characteristics, with a simulated energy resolution of $16 \%$ at $511 \mathrm{keV}$. Results refer to a lower energy window of 350-400 keV and an upper window of 500-550 keV, except where stated otherwise. Simulation inputs, outputs and scatter are 3-D; for purpose of presentation, only one slice is displayed.

\section{Energy Based Transmission Maximum Likelihood}

An energy based transmission tomography maximum likelihood (MLTR-EB) algorithm has been developed to estimate attenuation from scattered data. Reconstruction has been performed by relying on the L-BFGSB-B algorithm [16] (limited-memory Broyden-Fletcher-Goldfarb-Shanno with boundary constraints), which requires an explicit formulation of the $\log$-likelihood $\phi(\mu)$ and its gradient $\nabla \phi(\mu)$, which has been analytically derived from the forward model and then implemented in STIR. The implementation uses caching and other techniques in order to significantly reduce the computational time both in the likelihood and the gradient calculation. The gradient implementation has been validated with finite differences.

Starting from a guess $\mu_{0}$, the reconstruction algorithm attempts to recover a $\mu$-map which is as close as possible to $\mu^{\text {true }}$ :

$$
\mu=\underset{\mu \geq 0}{\arg \max } \phi(g \mid \lambda, \mu)
$$

where $\phi(g \mid \lambda, \mu)$ represents the log-likelihood function of the measured scattered data $g$.

At the moment the reconstruction algorithm uses segment 0 only, but it can be easily extended to other segments.

In the reconstruction simulations the lower energy window range has been set to $\mathrm{L}=350-460 \mathrm{keV}$ and an the upper energy window has been set to $\mathrm{U}=460-570 \mathrm{keV}$. A single energy window $\mathrm{O}=350-570 \mathrm{keV}$ has been also investigated as a comparison.

Poisson noise has been added to simulated data. 

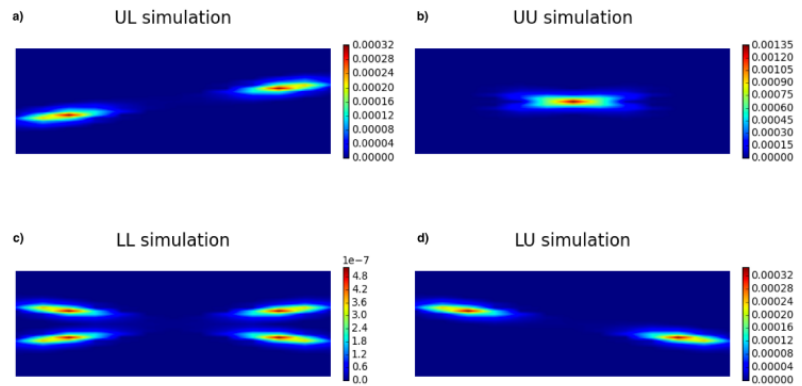

Fig. 3. Single scatter sinograms obtained with different energy window simulations. Note the different maxima used for color scale.

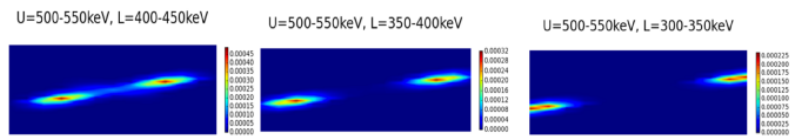

Fig. 4. Sinograms corresponding to three different lower energy windows.

\section{RESULTS}

\section{A. Point Source Simulations}

Fig. 3 visualizes scatter sinograms obtained for the point sources phantom. When the same energy window is used for both detectors, only a very small amount of counts is detected in the lower energy window LL (Fig. 3c), while the UU window (Fig. 3b), only detects scatter angles close to zero.

On the other hand, UL (Fig. 3a) and LU (Fig. 3d) outcomes shows two key advantages: the number of counts is significantly increased over LL, and two different sinograms can be obtained (with each giving spatial information on one of the emission point sources), indicating that some additional information on the annihilation position lies in scatter coincidences.

We also observed that by varying the lower energy window, it is possible to select counts from different scattering angles (Fig. 4).

\section{B. MLTR-EB Reconstruction}

The iterative reconstruction algorithm requires an initial guess of the attenuation map to estimate. Therefore, we firstly chose a uniform phantom as initialization image, which corresponds to the case where no anatomical information are available.

With a low level of noise (number of counts $=1.4 \mathrm{E}+08$ ) both LL and UL+LU seem to be able to estimate an attenuation map from scattered data, even though with a low spatial resolution (Fig. 5).

However, when initializing from the MRAC (no arms, 20\% lower lung attenuation coefficient), the reconstructed image shows detailed anatomical information as well as a higher spatial resolution.

The reconstruction method is also able to recover the arms, that were absent in the initialization and to compensate for errors due to the wrong assignment of population based density values in the lung.

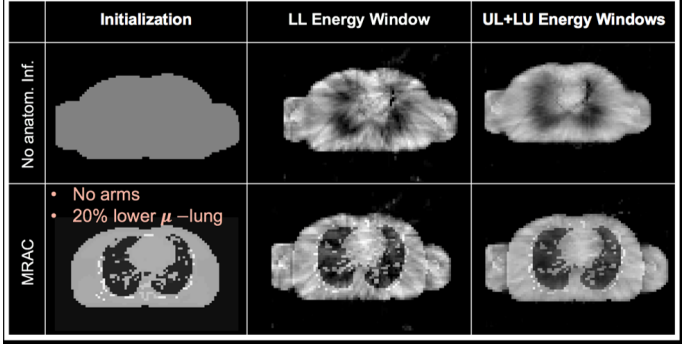

Fig. 5. Reconstructed attenuation map from different initialization for two energy window pairs. Low noise.

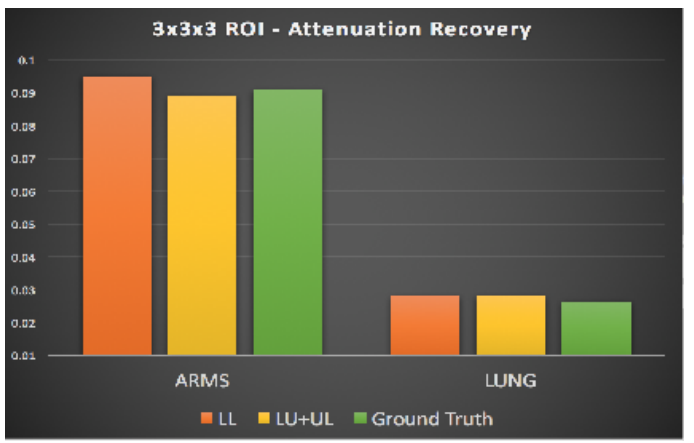

Fig. 6. Attenuation recovery in the arms and in the lung for LL and UL+LU energy window pairs.

We therefore investigated the attenuation recovery in two different regions of interest respectively in the lung and in the arms, and both energy window configuration seems to lead to quantitative accurate results (Fig. 6).

Simulations with a higher level of noise (number of counts $=1.4 \mathrm{E}+07$ ) show that the LL performance decreases tremendously, and this can be explained with the small amount of counts that this energy window pair detects.

By contrast, the UL+LU performance remains quite stable even in a high noise scenario (Fig. 7).

As a further investigation, we assessed the effects on quantification when accurate anatomical information are available. Fig. 8 shows results relative to ROIs respectively in the mediastinum and the lung.

Without any anatomical information, the estimated attenuation value overall approaches the ground truth over the iterations, and the UL+LU configuration (red curve) has the best performance. The LL pair (blue curve) remains quite far

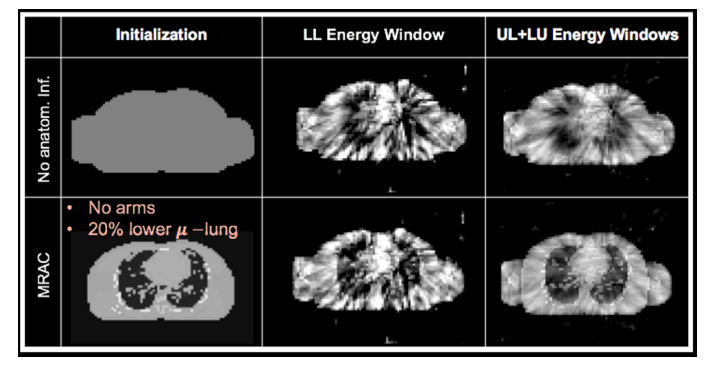

Fig. 7. Reconstructed attenuation map from different initialization for two energy window pairs. High noise. 


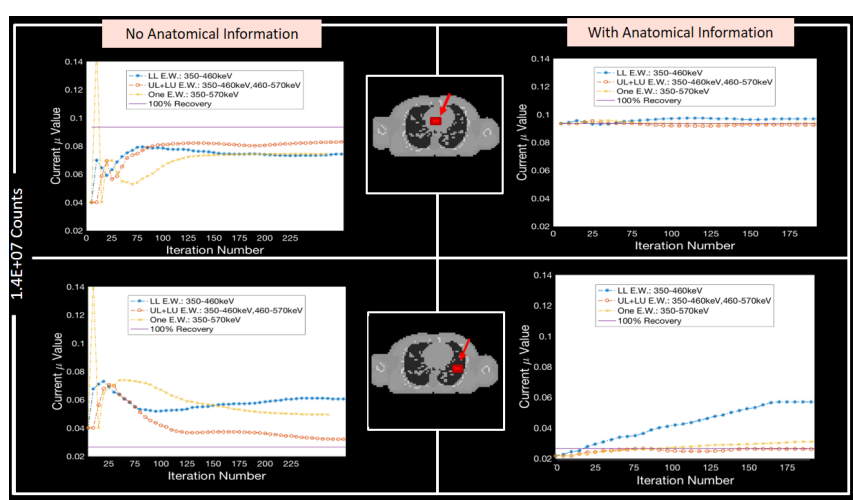

Fig. 8. Attenuation recovery in two different ROIs (mediastinum -first rowand lung -second row-),without anatomical information - first column, and with anatomical information - second column

from the ground truth, according to what has been shown with images. The single energy window (yellow curve) has been added as a comparison, and seems to have a similar performance to UL+LU.

When adding anatomical information, and therefore initializing from reasonable values, the reconstruction performance improves considerably.

\section{CONCLUSION}

Scatter coincidences yield information on the emission and attenuation images even when dealing with realistic energy measurements. Detection probability and SNR strongly depend on the energy window selection.

Results from simulations show that it is possible to obtain an attenuation map from scattered PET data only, albeit with a low spatial resolution. Adding MR information makes it possible to improve the spatial resolution and to see accurate anatomical features in the reconstructed image.

The current study is limited to single scatter only, whereby the lower energy window would likely have to be chosen to avoid too large contributions of multiple scatter at very low energies. We expect that multiple scatter events will contribute more to the lower energy window-pair (LL).

Another limitation of this study is that the simulation only included coincidence where one of the photons scattered. In practice, the presence of unscattered counts will need to be taken into account via a modification of the forward model and its gradient.

This work motivates incorporation of multiple energy window pairs into a joint emission and attenuation estimation algorithm as future work.

\section{ACKNOWLEDGEMENTS}

The authors would like to thank Dr. C.C. Watson (Siemens Healthineers) and Y.-J. Tsai (University College London) for helpful discussions.

\section{REFERENCES}

[1] J. Nuyts et al., "Simultaneous maximum a posteriori reconstruction of attenuation and activity distributions from emission sinograms," IEEE Trans. Med. Imaging, vol. 18, pp. 393-403, 1999.
[2] A. Rezaei et al., "ML-reconstruction for TOF-PET with simultaneous estimation of the attenuation factors," IEEE Trans. Med. Imaging, vol. 33, pp. 1563-1572, 2014.

[3] A. K. Shukla et al., "Positron emission tomography: An overview," $J$ Med Phys, vol. 31, no. 1, pp. 13-21, 2006.

[4] Y. Berker et al., "Use of scattered coincidences for emission-based estimation of attenuation map in PET,", IEEE NSS/MIC Conf. Rec, 2012.

[5] G. Zhang et al., "Feasibility of scatter based electron density reconstruction for attenuation correction in positron emission tomography,", IEEE NSS/MIC Conf. Rec, 2015.

[6] L. M. Popescu et al., "PET energy-based scatter estimation and image reconstruction with energy-dependent corrections," Phys Med Biol, vol. 51, no. 11, pp. 2919-37, 2006.

[7] S. C. Cade et al., "Use of measured scatter data for the attenuation correction of single photon emission tomography without transmission scanning," Med Phys, vol. 40, p. 082506 (12pp.), 2013.

[8] A. Bousse et al., "Joint Activity/Attenuation Reconstruction in SPECT Using Photopeak and Scatter Sinograms," IEEE NSS/MIC Conf. Rec, 2016.

[9] Y. Berker et al., "Scattered PET data for attenuation-map reconstruction in PET/MR," Med Phys, vol. 41, p. 102502 (13pp.), 2014.

[10] H. Sun et al., "Evaluation of the Feasibility and Quantitative Accuracy of a Generalized Scatter 2D PET Reconstruction Method," ISRN Biomedical Imaging, vol. 2013, p. 943051 (11 p), 2013.

[11] Y. Berker et al., "Numerical algorithms for scatter-to-attenuation reconstruction in PET: empirical comparison of convergence, acceleration, and the effect of subsets," IEEE NSS/MIC Conf. Rec, 2016.

[12] H. Sun et al., "A generalised scatter reconstruction algorithm for limited energy resolution PET detectors," IEEE NSS/MIC Conf. Rec, 2014.

[13] C. C. Watson et al., "A single scatter simulation technique for scatter correction in 3D PET," Three-Dimensional Image Reconstruction in Radiology and Nuclear Medicine, vol. 4, pp. 255-268, 1996.

[14] K. Thielemans et al., "STIR: Software for Tomographic Image Reconstruction Release 2," Physics in Medicine and Biology, vol. 57, no. 4, pp. 867-883, 2012.

[15] W. P. Segars et al., "4D XCAT phantom for multimodality imaging research," Med Phys, vol. 37, no. 9, pp. 4902-15, 2010.

[16] R. H. Byrd et al., "A Limited Memory Algorithm for Bound Constrained Optimization," SIAM Journal on Scientific and Statistical Computing, vol. 16, no. 5, pp. 1190-1208, 1995. 